\title{
DIE ERSTELLUNG VON VLOGMEDIEN ALS LERNMATERIAL FÜR DEN DEUTSCHUNTERRICHT
}

\author{
Tia Permata Sari \\ Ahmad Bengar Harahap \\ Risnovita Sari
}

\begin{abstract}
AUSZUG
Das Ziel dieser Untersuchung ist ein Vlogmedien Lernmaterial für den Deutschunterricht zu erstellen. In dieser Untersuchung wird die deskriptive qualitativeMethode angewendet. Diese Untersuchung wird in der Bibliothek der Fakultät für Sprache und Kunst der Staatlichen Universität von Medan durchgeführt. Der Prozess der Erstellung eines Vlogs Lernmaterial für den Deutschunterricht besteht aus der Erklärung der Phasen von ADDIE Theorie, sie sind: (1) die Analysephase, (2) die Designsphase, (3) die Erstellungsphase,(4) die Implementationsphase, und (5) die Evaluationsphase. Die Lernmaterial für den Deutschunterricht warden auf Deutsch und Indonesisch in einem Vlog vorgestellt. Der Name des Vlogs ist:

https://www.youtube.com/channel/UCoTGbGtxnKepx8S3toPxiJg?view_as=subsc riber. Es gibt 6 Posting, die in diesem Vlog publiziert werden, sie sind: Sich Vorstellen, Herkunft Wohnort und Sprachen, das Hobby, Schulsachen und Gegenstände, Was machst du in der Schule und Mein Tag. Die Evaluation der Erstellung desVlogs wird von den Experten gemacht. Die Ergebnisse der Evaluation zeigt, dass das Vlog gutist. Der Inhalt und Thema des Vlogs sind interessant zum Lernen. Die Darstellung ist deutlich. Das Vlogs ist interresant mit der vorhandene Unterteilung der Materialien. Die Materialien des Vlogs können die Lust den zuschauern wecken, um Deutsch zulernen.
\end{abstract}

Schlüsselwörter: Vlogs,Lernmaterial den Deutschunterricht

\section{EINLEITUNG}

Die Sprache ist ein Kommunikationsmittel. Ohne die Sprache kann man nicht kommunizieren. In der Globalisierung sollen die Leute Fremdsprachen beherrschen. Deutsch ist die zweite Sprache wichtigute in Europa, weil deutsch als Umgangsprache oder Unterrichtssprache benutzt wird, z.B in der Schweiz, Österreich und anderen Staaten in Europa (Milda Anika : 2013). Jetzt in Indonesien wird deutsch schon in der Schulewie im Gymnasium oder an der Universität unterrichtet. Während des Schulpratikumswollen viele Schüler klasse 
$\mathrm{X}$ nicht deutsch lernen.Die Schüler haben die schlechte Lernergebnisse. Die Benutzung von Lernmedien in der Schule reicht noch nicht aus und ist nicht interresant. In Wirklichkeit verwenden Lehrer nicht viele Medien. Daher haben die Schüler Schwierigkeiten beim Deutschlernen und die Schülerfühlender Lernprozess sehr langweilig.Also die Lehrer sollten neue Systeme für den Deutschunterricht haben. Der Lernprozess kann man mit Komputer und dem Internet unterstützt werden.Das Internet hat eine Medienrevolution eingeleitet. Ein Beispiel von Lernmedien ist Vlogmedium oder Videoblog. Die kürzliche Erweiterung des Blog zum Video-Format wird oft als Vlog bezeichnet, (Pilkington, 2009 : 3). Vlog ist ein vermittler audiovisuellen Inhalte im Internet. Die Anwendung des Vlog benutzen der Schüler für Verständnis Deutsch. Die Bemühungen können verschiedene Erfolgenhaben, so können Schüler eine neue Information im Lernprozess finden und das macht den Lernprozess interessanter. Das Vlogmedien können den Deutschuntterichtinteressanter gestalten, sodass die Schüler besser Lernmaterial deutsch verstehen. Vor diesem Hintergrund, interessiert sich die Verfasserin für die Forschung über Die Erstellung von Vlogmedien als Lernmaterial für den Deutschunterricht mit dem Subthemen Sich Vorstellen, Herkunft und Wohnort, das Hobby, Schulsachen und Gegenstände, was machst du in der Schule und mein Tag.

\section{THEORETISCHE GRUNDLAGE}

\section{Das Erstellungphase}

In dieser Untersuchung wird das ADDIE Modell benutzt.Asrar (2013:118) beschreiben, dass das ADDIE Model als Anleitung beim Aufbau der Vorrichtung erfüllt und Trainingsprogramme effektiv, dynamisch und unterstützt die Ausbildung. Es gibt fünf Phasen, um ein Interaktivmedium zum Lernen deutscher Grammatik zu entwickeln : 1) Die Analyse 2) Das Design 3) Die Erstellung 4) Die Implementation und 5) Die Evaluation

\section{Der Begriff des Lernmedium}

Das Lernmedium ist ein Vermittler des Lernsauftrags von Lernressourcen (der Lehrer) zum Empfänger (der Schüler), sodass die Interaktion zwischen Lehrer und Schüler erfolgen kann (Munir : 2008:5). Gagne (in Sadiman, 2008 :6) 
behauptet Medien sind die verschidenen Arten von Umweltkomponenten, die Schüler beim lernen unterstützen. Der Lernenprozess ist der Komunikationsprozess zwisschen Lehrern und Schülern, wozu der Lehrer das Medium als Lernressource benutzen kann . Das Medium liefert Informationen vom Lehrer zum Schüler. So kann geschlossen werden, des Medium eine Lieferung Lernmaterial vom Lehrer zum Schüler ist, sodass kann die Vernuft gereizt und wird die Aufmerksamkeit der Schüler im Lernprozess steigert.Es gibt zwei Arten von Lernmedien, das sind einfach Lernmedium und moderne Lernmedium. Das einfach Lernmedium ist z.B die Tafel, und das moderne Lernmedium sind z.B Komputer und Internet. Es gibt andere Arten von Lernmedien, wie das reale Objekt wie das Buch, Power Point, das Video, Audio und Computer-Software. Das Medium mit Bewegung ist das Video und Computer-Software und das Medium das nur mit Ton produzier ist das Audio in Form der Kasette oder als CD.

\section{Der Begriff des Vlog}

Ein Videoblog oder Vlog ist ein Webblog (Blog). Video istdie erste Quelle als das Medium nicht Text oder Audio. Vlog wurde im Jahr 2005 auf der Website Youtubebekannt . Mit der kürzliche Erweiterung wird der Blog im Video-Format oft als Vlogs bezeichnet (Pilkington : 2009 : 7). Das aktive bloggen mit Video nenntmanVlogging, Videocasting.Vlog ein audiovisuellen Vermittlen im Internet.Von vielen Pädagogen wird dem Vlog als potentielles Lernmedium gesehen.Es gibt einige Vorteile von Vlogmedien : a. Mit Vlogmedien kann mit dem Publikum direkt interagiert werden und über aktuelle Thema berichtet werden, b. Vlogmedien sind am besten für Tutorial Blogs geeignet. Im Blog kann das Publikum nur einen Text lesen, aber im Vlog kann der Herausgeber erklären. Deshalb ist Vlogmedien am besten für Video Turorials geeignet, c. Wenn das Video gut aussieht, gibt es die Möglichkeite viral zu gehen. Wenn es viral wird, wird das Video von vielen Menschen gesehen.Pocket Video ist die erste Applikation für Video-Blogging oder Vlogs auf der Welt. Mit Pocket Video können Vlog zu erstellen und Vlogs leicht bearbeiten und direkt in Youtube 
oderwo andere veröffentlichen. Außerdem, Pocket Video ist der einfachste und leistungsfähigste Video-Editor für jede Richtung.

\section{Youtube}

Youtube ist eine Populäre Video-Website. Youtube wurde im Februar 2005 von Chad Hurley, Steve Chen, und Jawed Karim gegründet. Im Jahr 2006, wurde Youtube von Google gekauft.Es gibt viele Arten von Vlogs auf Youtube, wie zu Ausbildung, täglichen Aktivitäten, alsTutorial, für Musik, usw. Viele Vlogger laden ihre Videos auf Youtube hoch.

\section{Der Begriff Lernmaterial}

Das Lernmaterial ist der Inhalt des Lehrplanszur Erreichung der Kompetenz in der Ausbildung. Das Lernmaterial kann sich auf drei verschiedene Aspekte beziehen : Kenntnisse, Fähigkeiten und die Haltung.Munir (2010:16) meint, dass Lernmaterial die Kenntnisse, die Haltung und die Fähigkeit enthält ist, die Schüler brauchen um Fähigkeiten oder Kompetenzen zu erlernen. Muslich (2010:193) meint, dass das Lernmaterial den Schülern als Haupwerkzeug dient, damit die Schüler die Grundkompetenzen erreichen. Laut den oben genannten Meinungen stellt das Lernmaterial die Grundlage des Lehrplans, durch den Kenntnisse und Fähigkeiten vermittelt werden, die von den Schülerinnen während des Lernprozesses gelernt werden müssen.Es gibt das Lernmaterial Klasse X von Jung Buch :

1. Subtheme Identität istVorstellen,Herkunft und Wohnort, Hobby.

2. Subtheme die Welt der Schule ist Schulsachen und Gegenstände,Was machst du in der Schule ?, Mein Tag.

\section{UNTERSUCHUNGSMETHODE}

Diese Untersuchung ist eine Erstellungsuntersuchung. Es wird Deutschlernmaterial erstellt. Diese Untersuchung benutzt das ADDIE Modell und die Deskriptive Methode.

\section{Die Daten und Die Datenquelle}

Die Datenquelle von dieser Untersuchung ist das Jung Buch mit den Subthemen (1) Sich Vorstellen, (2) Herkunft und Wohnort, (3) Das Hobby, (4) 
Schulsachen und Gegenstände, (5) Was machst du in der Schule, (6) Mein Tag. Die Daten stamen von einem Vlogs über Deutschlernen.

\section{Der Untersuchungsort}

Diese Untersuchung wird in der Bibliothek der Fakultät für Sprache und Kunst an der Staatlichen Universität von Medan durchgeführt.

\section{Die Skizze der Untersuchung}

Diese Untersuchung benutzt das Model von ADDIE.Dieser Theorie wird schon im Kapitel II erklärt. Des ADDIE Modell teilt sich in 5 Hauptschrittedes Erstellungsmodells, diese sind (1) die Analyse (2) dem Design (3) der Entwicklung (4) derUmsetzung und (5) der Evaluation.

\section{ERGEBNIS DER UNTERSUCHUNG}

\section{Der Prozess der Erstellung}

\section{Analysephase}

Bei der Analysephase werden die Berdüfnisse der Deutschschüler der SMAN 10 MEDAN bezüglich der Verwendung von Lernmedien im Lernprozess untersucht. Basierend auf den Observation ist bekannt, dass 2 Person von 10 Person der Schüler gute Noten haben. Bei dieser Untersuchung erstellte die Verfasserin eines Lernmaterial mit Vlogmedien, dass als Lernmedium für den Deutschunterricht verwendet wird. Die Verfasserin entwickelt das Thema "Sich Vorstellen, Herkunft Wohnort und Sprachen, das Hobby, Schulsachen und gegenstände, was machst du in der Schule ?, mein Tag” mit Vlogmedien. Mit Vlogmedien können Schüler eine neue Information im Lernprozess finden und das macht den Lernprozess interessanter. Das Vlogmedien können den Deutschunttericht interessanter gestalten, so dass die Schüler das Lernmaterial Deutsch besser verstehen.

\section{Designphase}

Zweite Phase ist die nächste Erklärung von der ersten Phase. Zuerst werden die Wörter entworfen, die Standort Viedeo zu machen gewählt und das Thema beschrieben. Danach werden die Vlogs Schriftart gewählt. In dieser 
Phase wird das Lernmedium mit den Vlogmedien erstellt.Es ergibt den Plan über das Vlog, das ist Thema, die Art der Buchstaben, und die Farbe.

\section{Erstellungsphase}

Das Konzept, dass in der zweite Phase geplant wird, wird in dieser Phase verwirklicht. In dieser Phase werden die Vlogmedien mit hilfe der PocketVideo Applikation erstellt

\section{Implementationphase}

In dieser Phase werden die Vlogmedien erstellt. Nun wird die Valiedierung gemacht. $\mathrm{Ob}$ die Vlogmedien schon gut sind oder verbessert werden müssen. Nach der Valiedierung werden die Vlogmedien verbessert. Diese Vlogmedien werden von Experten geprüft. Die Experten ist eine Komputer Dozentin an der Üniversität von Nord Sumatra, die über Medien Bescheid weiss. Ihr Name ist Elviwani S.Kom, M.Kom. Die Expertin hat die Daten schon korrigiert. Der Kommentar die Expertin ist sehr interessant. Die Expertin hat das Lernmedium benotet : Der Evaluationn von 10 kategorien der Aspekt der Evaluation gibt dei Expertin die Note 4 für acht Aspekt. Die Note 4 bedeutet sehr gut. Der Expertin die Note 3 für die Ubringe Aspekte. Es gibt 2 Aspekte. Basierend auf den Daten der Vlogmedien konnte dieses chon für der Schülern Klasse X verwendet werden.

\section{Evaluation}

Das ist die letzte Phase, um interessante Vlogmedien zu erstellen. Die Expertin werden die Rätschläge, Kommentare und die Bewertung angegeben. Der Kommentare von Expertin, dass das Vlogs können für Lernprozess benutzt werden. Der Ergebniss der Ton und Video wurden schon gut sind. Die Vlogmedien werden nicht notig Revision gemacht

\section{Das Ergebnis der Erstellung}

Nachdem das Design des Vlogs realisiert werden ist, werden die Daten im einem Vlog eingestellt. Bevor die Daten publiziert werden, können sie noch bearbeitet werden. Es wird gemacht um die gute Video oder Postings zu erhalten. 6 Postings werden bereits publiziert. Das Video ist die Rolle in Bibliothek von UNIMED, das Haus, und The Le Hu Garden gespielt 


\section{Diskussion}

Basierend auf den Ergebnissen der Erstellung des Vlogmedien, das mit der Hilfe Pocket Video Applikation zum Deutschlernen wurde zusammengefasst. In dieser Untersuchung wird die ADDIE Modell Theori benutzt. Diese Theori besteht aus fünf stufen nämlich :In dieser Kapitel wird jede stufe mit ihrem Ergebniss erklärt. In dieser Ermittlungsphase wird zuerst die Datensammlung und die Problemidentifizierung durchgeführt. In dieser zweite Phase wird das Konzept/Design des Vlogmedien mit der Pocket Video Applikation, um interessantes Vlogmedien zu erstellen. Das Design wird an das Thema angepasst, das in der Pocket Video Applikation integriert wird, um Schriftart und die Farbe Schriftart zu erstellen. Die nächste Phase ist Erstellungsphase. Das Lernmaterial wird mithilfe der Pocket Video Applikation entwickelt.Das Vlogmedien wurde schon mit Texten, und Ton erstellt.So dass interessantes Medium enthält. Die vierte Phase ist Implementationphase. Eine Expertin hat das Vlogmedien mit dem Pocket Video Applikation analysiert und korrigiert. Das Lernmedium bekommt die Note 3 über die Töne und die Texte.Die Töne und die Texte sind nicht ganzt klar. Die Verfasserin hat verbessert. Danach wird in der Implementation das erstellte Vlogmedien validiert. Basierend auf der Obigen Erklärung kann zusammengefasst werden, dass die Erstellung von Vlogmedien als Lernmaterial für den Deutschubnterricht sehr gut ist. Sodass die Lehrern die Schüler das Vlogmedien benutzen können.

\section{Schlussfolgerungen}

Nach den Untersuchungsergebnissen fallen die Schlussfolgerungen folgendermaßen aus :

1. Der Prozess der Erstellung von Vlogmedien als Lernmaterial für den Deutschunterricht. Dieser Prozess besteht aus der Erklärung der fünf Phasen von ADDIE-Modell. Darunter sind : (1) Analysephase (die Observation Lernergebniss), (2) Designphase (das Thema vorbereiten, die Art und die Farbe vorbereiten), (3) Erstellungphase (das Thema Vlogmedien mit PocketVideo entwickeln), (4) Implementationphase (Expertprüfen), (5) Evaluation (die Vlogmedien verbessert oder nicht). 
2. Das Ergebnis der Erstellung von Vlogmedien als Lernmaterial für den Deutschunterricht hergestellt. Sie sind nämlich :

a. Vlog 1,2,3 bestehen aus Thema Identität, mit dem Subthema sind Sich Vorstellen, Herkunft Wohnort und Sprachen, das Hobby. Gramatik sind Personalpronomen, und Kojugation das Verben. Das Ziel, die Schülern können sich selbst und andere über das Hobby einzuführen. Der Vlog gibt es Übungen. Vlog 1 dauert 13 Minuten, Vlog 2 dauert 12 Minuten, und Vlog 3 dauert 7 Minuten. Dieser Vlog ist für 1. Semester.

b. Vlog 4,5,6 bestehen aus Thema Schulleben, mit dem Subthema Schulsachen und Gegenstände, was machst du in der Schule und mein Tag. Gramatik sind die Artikel, Konjugation Verba haben, Nominativ und Akkusativ, Personalpronomen Akkusativ, Trennbare Verben und Konjugation Imperativsatz. Das Ziel, die Schülern können in der Lage zu beschreiben die Tätigkeiten ein Schüler, die Schülern können den Imperativsatz aussprechen, die Schülern können einen Satz mit Trennbare Verben aussprechen. Der Vlog gibt es Übungen. Vlog 4 dauert 14 Minuten, Vlog 5 dauert 16 Minuten und Vlog 6 dauert 13 Minuten. Dieser Vlog ist für 2. Semester. Der Vlog kann in Youtube sehen, mit dem Link :https://www.youtube.com/channel/UCoTGbGtxnKepx 8S3toPxiJg?vie w_as=subscriber. Dieses Linkist ein eigene Youtube Kanal.

\section{LITERATURVERZEICHNIS}

Aldoobre, Nada. 2015. ADDIE Model. University of Northen Colorado: American International Journal of Contenporary Research, V (6) 68-71

Hutabarat, Mery Dahlia. 2010. Jung Aktuell Band 1, Bandung: CV. Sarana Pustaka

Knowledge, Raf. 2010. Video Blogging untuk Pemula. Jakarta: Diperbanyak PT. Gramedia

Enterprise, Jubilee. 2008. Teknik Cepat Membuat Video Blog.Jakarta: PT Elex Media Komputindo 
Studia: Journal des Deutschsprogramms

p-ISSN 2301-6108

e-ISSN 2654-9573

https://jurnal.unimed.ac.id/2012/index.php/studia/index

Munir.2008. Kurikulum Berbasis Teknologi Informasi dan Komunikasi.Bandung:

Alfabeta

Pilkington, E. 2009.Merriam-Webster releases list of new words to be included in dictionary. London: The Guardian.

Pribadi, Benny. 2004. Desain dan Pengembangan Program Pelatihan Berbasis Kompetensi: Implementasi Model ADDIE. Jakarta: Kencana

Sadiman, Arief, dkk. 2008. Media Pendidikan: Pengertian, Pengembangan dan Pemanfaatannya. Jakarta: PT Raja Grafindo Persada

Setyosari, Punaji. 2010. Metode Penelitian Pendidikan dan Pengembangan.Jakarta: Kencana

Vertag, O’Really. 2010. Blogs, Video und Online-Journalismus. Köln: O’Reilly https://net.educause.edu/ir/library/pdf/ELI7005.pdf

http://statf.Uny.ac.id/sites/default/files/pengabdian/dra-endang-mulyatiningsih$\mathrm{mpd} / 7$ cpengembangan-model-pembelajaran.pdf

http://stil.uni-leipzig.de/wp-content/uploads/2015/04/advantages-disvantages.pdf http://www.academia.edu.edu/13294753/The_Effect_of_Vlogging_on_EFL_Stud ent_Teachers_Teaching_Self-efficacy 